\title{
Moduli mediation without moduli-induced gravitino problem
}

\author{
Kensuke Akita, ${ }^{a}$ Tatsuo Kobayashi, $^{b}$ Akane Oikawa $^{a}$ and Hajime Otsuka ${ }^{a}$ \\ ${ }^{a}$ Department of Physics, Waseda University, \\ Tokyo, 169-8555 Japan \\ ${ }^{b}$ Department of Physics, Hokkaido University, \\ Sapporo, 060-0810 Japan \\ E-mail: ken8a1@asagi.waseda.jp, kobayashi@particle.sci.hokudai.ac.jp, \\ a.oikawa@aoni.waseda.jp, h.otsuka@aoni.waseda.jp
}

ABSTRACT: We study the moduli-induced gravitino problem within the framework of the phenomenologically attractive mirage mediations. The huge amount of gravitino generated by the moduli decay can be successfully diluted by introducing an extra light modulus field which does not induce the supersymmetry breaking. Since the lifetime of extra modulus field becomes longer than usually considered modulus field, our proposed mechanism is applied to both the low- and high-scale supersymmetry breaking scenarios. We also point out that such an extra modulus field appears in the flux compactification of type II string theory.

KeYwords: Cosmology of Theories beyond the SM, Supergravity Models

ARXIV EPRINT: 1603.08399 


\section{Contents}

1 Introduction 1

2 Brief review of the moduli-induced gravitino problem in the mirage mediation

3 The dilution mechanism in $4 \mathrm{D} \mathcal{N}=1$ SUGRA $\quad 7$

4 The dilution mechanism in effective action of type II string theory 10

$4.1 \quad F$-term uplifting 11

4.1.1 Model $1 \quad 11$

4.1.2 Model $2 \quad 13$

4.1.3 Model $3 \quad 14$

$\begin{array}{lll}4.2 & \text { Uplifting with anti D-brane } & 15\end{array}$

$\begin{array}{ll}4.3 \text { The dilution mechanism } & 15\end{array}$

5 Conclusion $\quad 19$

A Moduli-dependent uplifting scenario 20

\section{Introduction}

Supersymmetry (SUSY) is a not only a phenomenologically plausible symmetry beyond the standard model (SM), but also is expected to appear in the low-energy effective theory of superstring theory. However, the lack of evidence of supersymmetry particles implies that the SUSY is broken above the TeV scale. In order to build in the SUSY-breaking scenario, we have to take care of the constraints from the collider experiments and cosmological observations, simultaneously. In particular, the mirage mediation [1-4], which is the mixture of modulus [5-8] and anomaly mediations [9, 10], predicts the characteristic sparticle spectrum in contrast to the other SUSY-breaking scenarios. The modulus mediation is achieved by the framework of Kachru-Kallosh-Linde-Trivedi (KKLT)-type moduli stabilization [11], where the volume modulus $T$ is stabilized at the AdS vacuum by the non-perturbative effects such as gaugino condensation on hidden D7-branes and Euclidean D-brane instanton, and the AdS vacuum is lifted to dS vacuum by anti D-branes. Since the $F$-term of $T$ is one-loop suppressed, the modulus mediation is comparable to the anomaly mediation. In this setup, the anomaly mediation and renormalization group effects cancel each other at a certain energy scale [3], and the pure modulus mediation appears at that energy scale. Such an energy scale is called as the mirage scale. In particular, the $\mathrm{TeV}$ scale mirage mediation is important, because one can relax the fine-tuning problem on the Higgs mass [12-14]. For example, in the next-to-minimal supersymmetric standard model with the $\mathrm{TeV}$ scale mirage mediation, one needs $\mathcal{O}(10) \%$ tuning for $1.5 \mathrm{TeV}$ gluino mass and $\mathcal{O}(1) \%$ fine-tuning even for several $\mathrm{TeV}$ of gluino mass to realize the weak scale $[15,16]{ }^{1}$

\footnotetext{
${ }^{1}$ See also ref. [17].
} 
In this paper, we focus on the cosmological aspects of pure modulus and mirage mediations. In the inflationary regime, the moduli fields are generically stabilized at the minimum away from those of KKLT-type moduli stabilization. This is because the moduli fields would receive the Hubble-induced masses due to the positive vacuum energy density or they couple to the inflaton field through the Planck-suppressed operators. Even if the moduli fields do not receive the Hubble-correction due to the shift symmetry, the quantum fluctuations deviate the minimum of moduli fields during the inflation. In any cases, when the Hubble scale is comparable to the masses of moduli fields, the moduli fields would oscillate around the true minima and such oscillating energy density dominates the energy density of the Universe. This is problematic from a cosmological point of view. First of all, the moduli fields should decay into the light particles before the start of Big Bang Nucleosynthesis (BBN) not to spoil the success of BBN. In addition, the moduli fields produce the huge amount of gravitinos. When the gravitino is unstable, the non-thermal lightest supersymmetric particle (LSP) is overproduced by the gravitino decay [18-20]. Since this moduli-induced gravitino problem occurs in both low-and high-scale SUSY-breaking scenarios, it motivates us to explore dilution of the gravitino abundance. There are several studies to dilute the overproduced LSP by the thermal inflation [21, 22], Q-ball [23] and unstable domain-wall [24], or the introduction of the axion sector [25]. The modulus oscillation may be suppressed by the adiabatic oscillation [26, 27]. Note that the heavier gravitino compared with the volume modulus is not relevant for the above moduli-induced gravitino problem, as can be shown in the large volume scenario [28, 29].

In this paper, we propose a new dilution mechanism by an inclusion of the extra light modulus field, which does not break the SUSY at the vacuum. In the framework of flux compactification of type IIB string theory on Calabi-Yau (CY) manifold, it was argued that on general grounds all the complex structure moduli and axion-dilaton are stabilized at the compactification scale [30]. However, it depends on the choice of three-form fluxes. We consider that one of the complex structure moduli remains massless under the flux compactification, and it can be stabilized by the instanton effects without breaking the SUSY. Since such complex structure modulus is lighter than Kähler modulus, it plays an important role of diluting the gravitino produced by the Kähler modulus. After briefly reviewing the cosmological aspects of mirage mediation, such as moduli-induced gravitino problem in section 2, we study the dilution mechanism based on the $4 \mathrm{D}$ effective $\mathcal{N}=1$ supergravity (SUGRA) in section 3 and the effective action of type II string theory in section 4, respectively. Finally, we conclude in section 5.

\section{Brief review of the moduli-induced gravitino problem in the mirage mediation}

We start with the 4D SUGRA originating from the type IIB string theory on CY orientifold. The moduli Kähler potential is described in the reduced Planck unit, ${ }^{2}$

$$
\begin{aligned}
K= & -3 \ln (-i(T-\bar{T}))-\ln (-i(\tau-\bar{\tau})) \\
& -\ln \left[2 i(F-\bar{F})-i\left(U^{i}-\bar{U}^{i}\right)\left(\partial_{i} F+\partial_{\bar{i}} \bar{F}\right)\right],
\end{aligned}
$$

\footnotetext{
${ }^{2}$ In this paper, we work the reduced Planck unit $M_{\mathrm{Pl}}=2.4 \times 10^{18} \mathrm{GeV}$, unless we specify it.
} 
where $T$ is the simplified overall Kähler modulus, $\tau$ is the axion-dilaton, and $F$ is the prepotential as functions of complex structure moduli $U^{i}\left(i=1,2, \cdots, h^{1,2}\right)$ with $h^{1,2}$ being the hodge number of CY manifold. The background three-form fluxes allow us to stabilize all the complex structure moduli and axion-dilaton with the following superpotential [31],

$$
W=\sum_{\alpha=1}^{2 h^{1,2}+2}\left(f^{\alpha}-\tau h^{\alpha}\right) \Pi_{\alpha},
$$

where $f^{\alpha}\left(h^{\alpha}\right)$ denotes the quanta of three-form fluxes relevant for the Ramond (NeveuSchwarz) sector, and $\Pi_{\alpha}$ is the period vector of CY manifold determined by the prepotential $F$. Below the mass scales of stabilized complex structure moduli and axion-dilaton, we can extract the effective potential of Kähler modulus. Within the framework of KKLT moduli stabilization, the Kähler potential and superpotential for overall Kähler modulus $T$ read as

$$
\begin{aligned}
K & =-3 \ln (-i(T-\bar{T}))+\text { const. } \\
W & =w_{0}+A e^{i a T}
\end{aligned}
$$

where $w_{0}$ is the real constant determined by the vacuum expectation values of the stabilized moduli, and the second term of superpotential is genereted by non-perturbative effects, e.g., gaugino condensation on $\mathrm{SU}(N)$ pure super Yang-Mills theory living on hidden D7-brane with $a=8 \pi^{2} / N$ and $A$ being real constant. By representing the chiral superfields as their scalar components in the same notation, the stabilization condition of overall volume modulus, $T=T_{\mathcal{R}}+i T_{\mathcal{I}}$, is given by

$$
\begin{aligned}
\left\langle T_{\mathcal{I}}\right\rangle & \sim \frac{1}{a} \ln \left(-\frac{2 A}{3 w_{0}}\right), \\
\left\langle T_{\mathcal{R}}\right\rangle & =0
\end{aligned}
$$

which leads to the AdS vacuum. After uplifting the AdS vacuum to dS vacuum, the Kähler modulus obtains the $F$-term, $F^{T}=-e^{K / 2} K^{T \bar{T}}\left(\partial_{\bar{T}} \bar{W}+K_{\bar{T}} \bar{W}\right)$ with $K^{T \bar{T}}$ being the inverse of Kähler metric $K_{T \bar{T}}=\partial_{T} \partial_{\bar{T}} K$, written in the gravitino mass $m_{3 / 2}=e^{\langle K / 2\rangle}\langle W\rangle$,

$$
\left\langle\frac{F^{T}}{T-\bar{T}}\right\rangle \simeq \frac{3 m_{3 / 2}}{2 a T_{\mathcal{I}}}
$$

which is comparable to the anomaly mediation $[9,10]$ in the case of $a\left\langle T_{\mathcal{I}}\right\rangle=\mathcal{O}\left(4 \pi^{2}\right)$,

$$
\frac{1}{16 \pi^{2}}\left\langle\frac{F^{C}}{C_{0}}\right\rangle \simeq \frac{m_{3 / 2}}{16 \pi^{2}}+\frac{\left(\partial_{T} K\right) F^{T}}{16 \pi^{2}}
$$

where $C=C_{0}+\theta^{2} F^{C}$ denotes the chiral compensator. When the Kähler modulus is stabilized at the racetrack minimum $[32,33]$ or it couples to the SUSY-breaking field in the $F$-term uplifting scenario [34-38], it is possible to change the ratio of anomaly to modulus mediation. (See for more details, e.g., refs. [35-38].) This results in the characteristic pattern of supersymmetric spectra without requiring the severe tuning. 
The mirage mediation is theoretically and phenomenologically attractive scenario. Here, let us revisit cosmological aspects of the mirage mediation. During the inflationary era, the moduli fields generically stay at the minimum away from that of KKLT-type moduli stabilization by the Hubble-induced mass. Thus, when the Hubble scale becomes comparable to the supersymmetric modulus mass, the modulus field rolls down to its true minimum and oscillates around it. Such oscillating energy dominates the energy density of the Universe. The total decay rate of modulus field is mostly captured by the modulus decay into gauge boson and gaugino pairs. The relevant Lagrangian density is described by

$$
\mathcal{L}=\int d^{2} \theta\left[\sum_{a=1}^{3} \frac{f_{a}}{4} W^{a \alpha} W_{\alpha}^{a}+\text { h.c. }\right],
$$

where $W_{a \alpha}$ are the gauge field strength superfields with $a=1,2,3$ being the standard model gauge group, $\mathrm{U}(1)_{Y}, \mathrm{SU}(2)_{L}, \mathrm{SU}(3)_{C}$, and $f_{a}$ are the gauge kinetic functions. When the standard model gauge group is derived from a single stack of D7-branes, the gauge kinetic function is given by

$$
f_{a}=k T+\Delta f_{a}(\langle U\rangle,\langle S\rangle)
$$

where $k$ is constant and $\Delta f_{a}$ are heavy moduli-dependent threshold corrections. The decay width of real and imaginary parts of moduli into gauge boson and gaugino pairs are the same as each other,

$$
\Gamma_{\text {tot }}^{T}=\sum_{a=1}^{3} \frac{N^{a}}{64 \pi}\left(\frac{|k| g_{a}^{2}}{\sqrt{\left\langle K_{T \bar{T}}\right\rangle}}\right)^{2} \frac{m_{T}^{3}}{M_{\mathrm{Pl}}^{2}} \simeq 2.9 \times 10^{-21}|k|^{2}\left\langle K^{T \bar{T}}\right\rangle\left(\frac{m_{T}}{10^{6} \mathrm{GeV}}\right)^{3} \mathrm{GeV}
$$

with $N^{a}=\{8,3,1\}$ and $\left(g_{a}\right)^{2} \simeq 0.53$ be the gauge couplings at the grand unification scale in the case of minimal supersymmetric standard model (MSSM), which gives rise to a radiation-dominated Universe with a reheating temperature,

$$
T_{\text {reh }}^{T} \simeq\left(\frac{\pi^{2} g_{*}\left(T_{\text {reh }}^{T}\right)}{90}\right)^{-1 / 4} \sqrt{M_{\mathrm{Pl}} \Gamma_{\text {tot }}^{T}} \simeq 80|k|\left\langle K^{T \bar{T}}\right\rangle^{1 / 2}\left(\frac{m_{T}}{10^{6} \mathrm{GeV}}\right)^{3 / 2} \mathrm{MeV} .
$$

Here, $g_{*}\left(T_{\text {reh }}^{T}\right) \simeq 10.75$ represents the effective number of degrees of freedom at the temperature $T_{\text {reh }}^{T}$.

In addition, the gravitino is also generated by the modulus decay with the following Lagrangian density in the unitary gauge,

$$
\begin{aligned}
\mathcal{L}_{3 / 2}= & -\frac{\epsilon^{\mu \nu \rho \sigma}}{2} \bar{\Psi}_{\mu} \gamma_{5} \gamma_{\nu} \partial_{\rho} \Psi_{\sigma}+\frac{\epsilon^{\mu \nu \rho \sigma}}{8}\left(\left\langle G_{T}\right\rangle \partial_{\rho} \tilde{T}-\left\langle G_{\bar{T}}\right\rangle \partial_{\rho} \overline{\tilde{T}}\right) \bar{\Psi}_{\mu} \gamma_{\nu} \Psi_{\sigma} \\
& -\frac{1}{4} m_{3 / 2} \bar{\Psi}_{\mu}\left[\gamma^{\mu}, \gamma^{\nu}\right] \Psi_{\nu}-\frac{1}{8} m_{3 / 2}\left(\left\langle G_{T}\right\rangle \tilde{T}+\left\langle G_{\bar{T}}\right\rangle \overline{\tilde{T}}\right) \bar{\Psi}_{\mu}\left[\gamma^{\mu}, \gamma^{\nu}\right] \Psi_{\nu}
\end{aligned}
$$

where $\Psi_{\mu}$ denotes the gravitino in the four-component formalism, $\tilde{T}=T-\langle T\rangle$, and $G_{T}=\partial G / \partial T$ with $G=K+\ln |W|^{2}$. We find that the decay width from the canonically 
normalized inflaton into the gravitino pair is

$$
\begin{aligned}
\Gamma_{3 / 2}^{T} & \simeq \frac{1}{288 \pi\left\langle K_{T \bar{T}}\right\rangle}\left|\left\langle\frac{D_{T} W}{W}\right\rangle\right|^{2} \frac{m_{T}^{5}}{m_{3 / 2}^{2} M_{\mathrm{Pl}}^{2}} \\
& =\frac{\left|\left\langle K^{T \bar{T}}\right\rangle\right|^{2}}{96 \pi}\left\langle\frac{F^{T}}{T-\bar{T}}\right\rangle^{2} \frac{m_{T}^{5}}{m_{3 / 2}^{4} M_{\mathrm{Pl}}^{2}}
\end{aligned}
$$

with $D_{T} W=\partial_{T} W+\left(\partial_{T} K\right) W$.

Let us denote the branching ratio from modulus to gravitino as $B_{3 / 2}$ which is typically of $\mathcal{O}(0.01-0.1)$ as pointed out in ref. [18]. Then, the gravitino yield which is the ratio of number density of gravitino to entropy density of the Universe, is estimated as

$$
Y_{3 / 2} \equiv \frac{n_{3 / 2}}{s} \simeq 2 B_{3 / 2} \frac{3 T_{\mathrm{reh}}^{T}}{4 m_{T}} \simeq 1.2 \times 10^{-7} B_{3 / 2}|k|\left\langle K^{T \bar{T}}\right\rangle^{1 / 2}\left(\frac{m_{T}}{10^{6} \mathrm{GeV}}\right)^{1 / 2},
$$

which is preserved until the gravitino decays. When the non-thermally produced gravitinos decay into all the MSSM particles with the decay width $\Gamma_{3 / 2}=193 m_{3 / 2}^{3} /\left(384 \pi M_{\mathrm{Pl}}^{2}\right)$, the decay temperature of gravitino becomes

$$
\begin{aligned}
T_{3 / 2} & \simeq\left(\frac{90}{\pi^{2} g_{*}\left(T_{3 / 2}\right)}\right)^{1 / 4} \sqrt{\Gamma_{3 / 2} M_{\mathrm{Pl}}} \\
& \simeq 7.8\left(\frac{10.75}{g_{*}\left(T_{3 / 2}\right)}\right)^{1 / 4}\left(\frac{m_{3 / 2}}{10^{5} \mathrm{GeV}}\right)^{3 / 2} \mathrm{MeV} .
\end{aligned}
$$

Note that the gravitino does not dominate the Universe at the decay of gravitino, since the energy density of the gravitino $\rho_{3 / 2}$ is not larger than that of radiation $\rho_{r}$,

$$
\frac{\rho_{3 / 2}}{\rho_{r}} \simeq \begin{cases}\frac{T_{\mathrm{NR}}}{T_{3 / 2}} B_{3 / 2}<1 & \left(T_{\mathrm{NR}}>T_{3 / 2}\right) \\ B_{3 / 2}<1 & \left(T_{\mathrm{NR}}<T_{3 / 2}\right)\end{cases}
$$

where we denote the nonrelativistic temperature of gravitino as $T_{\mathrm{NR}} \simeq\left(m_{3 / 2} / m_{T}\right) T_{\mathrm{reh}}^{T}$.

Finally, the dark matter is generated from the gravitino decay. Here and in what follows, we assume that the dark matter is consisted of the LSP under the assumption of $R$-parity conservation. The relic abundance of the dark matter, $Y_{\mathrm{LSP}}$, is found by solving the Boltzmann equation [39],

$$
Y_{\mathrm{LSP}}^{-1}=\left.Y_{3 / 2}^{-1}\right|_{T=T_{3 / 2}}+\left.\left(\sqrt{\frac{45}{8 \pi^{2} g_{*}\left(T_{3 / 2}\right)}} \frac{1}{M_{\mathrm{Pl}} T_{3 / 2}\left\langle\sigma_{\mathrm{ann}} v\right\rangle}\right)^{-1}\right|_{T=T_{3 / 2}},
$$

where $T_{3 / 2}$ is the decay temperature of gravitino and $\left\langle\sigma_{\mathrm{ann}} v\right\rangle$ is the thermally averaged annihilation cross section of the dark matter.

Now we take a closer look at the cosmological aspects of mirage mediation in the light of gravitino mass. When the gravitino mass is of $\mathcal{O}(30) \mathrm{TeV}$, the gravitino spoils the successful BBN unless $Y_{3 / 2}$ must be smaller than $\mathcal{O}\left(10^{-12}\right)$ [40-42]. Thus, eq. (2.13) implies 
that the branching ratio, $B_{3 / 2}$, should be smaller than $\mathcal{O}\left(10^{-5}\right)$. Even if the gravitino mass is larger than $\mathcal{O}(30) \mathrm{TeV}$, the dark matter abundance produced by the gravitino decay is overabundant to the Planck result as shown later.

First we take up the low-scale SUSY-breaking scenario, where the mass of LSP is of $\mathcal{O}(100) \mathrm{GeV}$. When the annihilation of the LSPs produced by the gravitino is not effective, the first term in eq. (2.15) dominates and the dark matter abundance is approximately given by

$$
\Omega_{\mathrm{LSP}} h^{2} \simeq m_{\mathrm{LSP}} Y_{3 / 2} \frac{s_{\text {now }}}{\rho_{\mathrm{cr}}} \simeq 3342 B_{3 / 2}|k|\left\langle K^{T \bar{T}}\right\rangle^{1 / 2}\left(\frac{m_{\mathrm{LSP}}}{100 \mathrm{GeV}}\right)\left(\frac{m_{T}}{10^{6} \mathrm{GeV}}\right)^{1 / 2},
$$

where $\rho_{\text {cr }} / s_{\text {now }} \simeq 3.6 h^{2} \times 10^{-9}$ is the ratio of critical density to the current entropy density of Universe, and $h$ is the dimensionless Hubble parameter. Here, we suppose that the annihilation cross section of LSPs is of $\mathcal{O}\left(10^{-7}-10^{-8} \mathrm{GeV}^{-2}\right)$, which is consistent with those of wino-like neutralino [39],

$$
\left\langle\sigma_{\mathrm{ann}} v\right\rangle \simeq \frac{g_{2}^{4}}{2 \pi} \frac{m_{\mathrm{LSP}}^{2}}{\left(2 m_{\mathrm{LSP}}^{2}-m_{W}^{2}\right)^{2}}\left(1-\frac{m_{W}^{2}}{m_{\mathrm{LSP}}^{2}}\right)^{3 / 2},
$$

with $m_{W}$ being the $W$-boson mass, and Higgsino-like neutralino into the $W$-boson pair [43],

$$
\left\langle\sigma_{\mathrm{ann}} v\right\rangle \simeq \frac{g_{2}^{4}}{32 \pi} \frac{m_{\mathrm{LSP}}^{2}}{\left(2 m_{\mathrm{LSP}}^{2}-m_{W}^{2}\right)^{2}}\left(1-\frac{m_{W}^{2}}{m_{\mathrm{LSP}}^{2}}\right)^{3 / 2},
$$

in the absence of co-annihilation effect. Therefore, the dark matter abundance in the lowscale SUSY-breaking scenario is overabundant to the Planck result, $0.1175 \leq \Omega_{\mathrm{LSP}} h^{2} \leq$ $0.1219[44,45]$, unless the branching ratio is smaller than $\mathcal{O}\left(10^{-4}\right)$.

On the other hand, in the high-scale SUSY-breaking scenario, the second term in eq. (2.15) dominates the LSP yield. The dark matter abundance is then given by

$$
\begin{aligned}
\Omega_{\mathrm{LSP}} h^{2} & \simeq m_{\mathrm{LSP}} \sqrt{\frac{45}{8 \pi^{2} g_{*}\left(T_{3 / 2}\right)}} \frac{1}{M_{\mathrm{Pl}} T_{3 / 2}\left\langle\sigma_{\mathrm{ann}} v\right\rangle} \frac{s_{\mathrm{now}}}{\rho_{\mathrm{cr}}} \\
& \simeq 65\left(\frac{80}{g_{*}\left(T_{3 / 2}\right)}\right)^{1 / 2}\left(\frac{10^{6} \mathrm{GeV}}{m_{3 / 2}}\right)^{3 / 2}\left(\frac{m_{\mathrm{LSP}}}{1 \mathrm{TeV}}\right)^{3}\left(\frac{10^{-3} \mathrm{GeV}^{-2}}{m_{\mathrm{LSP}}^{2}\left\langle\sigma_{\mathrm{ann}} v\right\rangle}\right),
\end{aligned}
$$

where the dark matter is assumed to be the wino-like or Higgsino-like neutralinos. Since, in the gravity-mediated SUSY-breaking scenario, the mass of LSP increases as a consequence of large gravitino mass, the overabundance of dark matter is common feature in the modulidominated Universe.

So far, there are several studies to dilute the gravitino abundance via the thermal inflation [22], Q-ball [23] and unstable domain-wall [24] or the introduction of the axion sector [25]. The modulus oscillation may be suppressed by the adiabatic oscillation $[26,27]$. In the next sections 3 and 4, we show the new dilution mechanism by introducing an extra chiral multiplet. 


\section{The dilution mechanism in $4 \mathrm{D} \mathcal{N}=1$ SUGRA}

Here, we propose a new dilution mechanism based on the $4 \mathrm{D} \mathcal{N}=1$ SUGRA. To reduce the gravitino abundance by the modulus decay, we introduce another chiral multiplet $\Phi$ with the following ansatz of (real) Kähler potential and superpotential, ${ }^{3}$

$$
\begin{aligned}
K & =-3 \ln (-i(T-\bar{T}))+K(\Phi, \bar{\Phi}), \\
W & =w_{0}+A e^{i a T} .
\end{aligned}
$$

In terms of the above Kähler potential and superpotential, the $F$-term scalar potential on the basis of $4 \mathrm{D} \mathcal{N}=1$ SUGRA is described by

$$
V=e^{K}\left(K^{I \bar{J}} D_{I} W D_{\bar{J}} \bar{W}-3|W|^{2}\right),
$$

where $D_{I} W=W_{I}+K_{I} W, K_{I}=\partial K / \partial \Phi^{I}, W_{I}=\partial W / \partial \Phi^{I}$ with $\Phi^{I}=T$, $\Phi$, and $K^{I \bar{J}}$ is the inverse of the Kähler metric $K_{I \bar{J}}$. Since there is no kinetic mixing between $T$ and $\Phi$, they are independently stabilized at the supersymmetric AdS minimum satisfying

$$
\begin{aligned}
& D_{T} W=0, \\
& D_{\Phi} W=K_{\Phi} W=0 .
\end{aligned}
$$

To uplift the AdS vacuum to the dS vacuum with tiny cosmological constant, we require the certain uplifting scenario as discussed in the next section. If we assume that the uplifting sector does not depend on the added chiral superfield $\Phi, \Phi$ still stays at the supersymmetric minimum given by eq. (3.3). In this supersymmetric minimum, the mass squared of modulus field is found in the limit of $a\left\langle T_{\mathcal{I}}\right\rangle \gg 1$,

$$
m_{T}^{2} \simeq \frac{\partial_{T} \partial_{\bar{T}} V}{K_{T \bar{T}}} \simeq 4\left(a\left\langle T_{\mathcal{I}}\right\rangle\right)^{2} m_{3 / 2}^{2}
$$

whereas the mass matrix of $\Phi=\Phi_{\mathcal{R}}+i \Phi_{\mathcal{I}}$ is given by

$$
\begin{aligned}
m_{\Phi}^{2}= & \left(2 K_{\Phi \bar{\Phi}}\right)^{-1}\left(\begin{array}{cc}
\partial_{\Phi_{\mathcal{R}}} \partial_{\Phi_{\mathcal{R}}} V & \partial_{\Phi_{\mathcal{R}}} \partial_{\Phi_{\mathcal{I}}} V \\
\partial_{\Phi_{\mathcal{R}}} \partial_{\Phi_{\mathcal{I}}} V & \partial_{\Phi_{\mathcal{I}}} \partial_{\Phi_{\mathcal{I}}} V
\end{array}\right) \\
& =\frac{m_{3 / 2}^{2}}{4}\left(K^{\Phi \bar{\Phi}}\right)^{2}\left(\begin{array}{cc}
4\left|K_{\Phi \Phi_{\mathcal{R}}}\right|^{2} & \left(K_{\Phi_{\mathcal{R}} \Phi_{\mathcal{R}}}+K_{\Phi_{\mathcal{I}} \Phi_{\mathcal{I}}}\right) K_{\Phi_{\mathcal{R}} \Phi_{\mathcal{I}}} \\
\left(K_{\Phi_{\mathcal{R}} \Phi_{\mathcal{R}}}+K_{\Phi_{\mathcal{I}} \Phi_{\mathcal{I}}}\right) K_{\Phi_{\mathcal{R}} \Phi_{\mathcal{I}}} & 4\left|K_{\Phi \Phi_{\mathcal{I}}}\right|^{2}
\end{array}\right) .
\end{aligned}
$$

When the kinetic mixing between the real and imaginary parts of $\Phi$ is absent at the minimum, i.e., $\left\langle K_{\Phi_{\mathcal{R}} \Phi_{\mathcal{I}}}\right\rangle=0$, we always obtain the positive mass squared of $\Phi$. Furthermore, with $\mathcal{O}(1)$ value of Kähler metric, $K_{\Phi \bar{\Phi}}=\mathcal{O}(1)$, the modulus mass is typically larger than that of $\Phi$,

$$
m_{\Phi_{\mathcal{R}}}^{2} \simeq m_{\Phi_{\mathcal{I}}}^{2} \simeq m_{3 / 2}^{2}
$$

\footnotetext{
${ }^{3}$ Although $\Phi$ is identified as the complex structure modulus field in the next section, the extension to other models are straightforward.
} 
We remark that the above positive mass squared of $\Phi$ cannot be realized at the AdS minimum without the uplifting sector. (See for ref. [46] in the situation that $\Phi$ corresponds to the no-scale modulus.)

Let us take a closer look at the dynamics of light added field $\Phi$. As we mentioned above, the modulus field $T$ gravitationally couples to the matter fields and then the minimum of modulus field during the inflation, $T_{1}$, is generically different from the minimum given in eq. (3.3), which are represented by $T_{0}$ and $\Phi_{0}$ in what follows. Moreover, the gravitational interaction between $T$ and inflaton sector induces the Planckian distance between $T_{1}$ and $T_{0}$. When the Hubble scale $H$ is comparable to the mass scale of $T, m_{T}$, modulus field rolls down to the true minimum and dominates the energy density of the Universe as mentioned in section 2. In a way similar to the modulus field, we consider that the added field $\Phi$ only gravitationally couples to the matter fields on the same footing.

Such a situation is captured by the following simplified scalar potential, ${ }^{4}$

$$
V_{\mathrm{osc}}=\frac{H^{2}(t)}{2}\left|T-T_{1}\right|^{2}+\frac{m_{T}^{2}}{2}\left|T-T_{0}\right|^{2}+\frac{H^{2}(t)}{2}\left|\Phi-\Phi_{1}\right|^{2}+\frac{m_{\Phi}^{2}}{2}\left|\Phi-\Phi_{0}\right|^{2},
$$

where $T_{1}$ and $\Phi_{1}$ denote the minimum induced by the Hubble parameter $H(t)$. Here, we denote the canonically normalized fields of $T$ and $\Phi$ as the same notation $T$ and $\Phi$, respectively. Since, in our model, the added field $\Phi$ is lighter than the modulus $T, \Phi$ oscillates subsequent to the oscillation of $T$, i.e., $t_{\mathrm{osc}}^{\Phi}>t_{\mathrm{osc}}^{T}$, where the oscillating time of scalar fields $\Phi$ and $T$ are defined as $t_{\mathrm{osc}}^{\Phi}$ and $t_{\mathrm{osc}}^{T}$, respectively. In the regime $t_{\mathrm{osc}}^{T}<t<t_{\mathrm{osc}}^{\Phi}$, the equation of motion of $T$ reads,

$$
\begin{aligned}
\frac{d^{2} T}{d t^{2}}+3 H \frac{d T}{d t}+m_{T}^{2}\left(T-T_{0}\right) & =0 \\
3 H^{2} & \simeq \frac{1}{2}\left|\frac{d T}{d t}\right|^{2}+\frac{m_{T}^{2}}{2}\left|T-T_{0}\right|^{2} .
\end{aligned}
$$

When we redefine the modulus field as $\tilde{T}=a^{3 / 2}\left(T-T_{0}\right)$, eq. (3.8) is rewritten as

$$
\frac{d^{2} \tilde{T}}{d t^{2}}+\left(m_{T}^{2}-\frac{3}{2} \frac{d H}{d t}-\frac{9}{4} H^{2}\right) \tilde{T}=0
$$

which can be solved under $m_{T}>\{H,|d H / d t|\}$,

$$
\tilde{T}(t)=\tilde{T}_{0} \sin \left(m_{T} t\right),
$$

with $\tilde{T}_{0}$ being constant. Furthermore, the virial theorem, $\frac{1}{2}\left\langle\frac{d T}{d t}\right\rangle^{2}=\frac{1}{2}\left\langle m^{2} T^{2}\right\rangle=a^{-3} m^{2} \tilde{T}_{0}^{2} / 4$, enables us to solve the time evolution of modulus field $T(t)$,

$$
T(t) \simeq \sqrt{\frac{8}{3}} \frac{M_{\mathrm{Pl}}}{m_{T} t} \sin \left(m_{T} t\right)
$$

from which the initial displacement $\Delta T=T_{1}-T_{0}$ is assumed to be of $\mathcal{O}\left(M_{\mathrm{Pl}}\right)$ at the time $t_{\mathrm{osc}}^{T} \simeq 1 / m_{T}$. The light field $\Phi$ also oscillates at the time $t_{\mathrm{osc}}^{\Phi} \simeq 1 / m_{\Phi}$ in a similar fashion.

\footnotetext{
${ }^{4}$ We do not consider the adiabatic suppression scenario where the coefficient of Hubble parameter is much larger than unity [26, 27].
} 
When we assume that the initial displacement of $\Phi$ as $\Delta \Phi=\mathcal{O}\left(M_{\mathrm{Pl}}\right)$, the oscillating energy densities of $\Phi$ and $T$ at the time $t_{\mathrm{osc}}^{\Phi} \simeq 1 / m_{\Phi}$ are almost the same as each other,

$$
\frac{1}{2} m_{T}^{2} T\left(t_{\mathrm{osc}}^{\Phi}\right)^{2} \simeq \frac{1}{2} m_{\Phi}^{2}(\Delta \Phi)^{2}
$$

It implies that two scalar fields dominate the energy densities of the Universe at the time $t_{\mathrm{osc}}^{\Phi}$.

Next, let us move onto the reheating process of $T$ and $\Phi$. As shown in eq. (2.9), the modulus field dominantly decays into the gauge boson pairs. By contrast, although we do not determine the couplings between $\Phi$ and standard model sector, for the time being, we assume that the decay temperature of $\Phi$ is much smaller than that of modulus field $T$. (We will provide the detailed setup in the next section.) To simplify our analysis, we further assume that decay temperatures of real and imaginary parts of $\Phi$ are the same. Therefore, the lightest field, $\Phi$, dominates the energy density of the Universe at the time later than the decay time of $T, t_{\mathrm{dec}}^{T} \simeq 1 / \Gamma_{\mathrm{tot}}^{T} \cdot{ }^{5}$ Keeping mind that $\Phi$ has the vanishing $F$-term, we find that this field does not decay into the gravitino(s). At the decay time of $\Phi, t_{\mathrm{dec}}^{\Phi} \simeq 1 / \Gamma_{\text {tot }}^{\Phi}$ with $\Gamma_{\text {tot }}^{\Phi}$ being the total decay width of $\Phi$, the produced entropy dilutes the gravitino (and LSP) yield given through the decay of $T$ at the time $t_{\mathrm{dec}}^{T}$. The dilution factor defined by the ratio between the entropy density of $\Phi\left(s_{\Phi}\right)$, and that of $T\left(s_{T}\right)$, at the time $t_{\mathrm{dec}}^{\Phi}$ is given by

$$
\begin{aligned}
\Delta_{S} & \simeq \frac{s_{\Phi}\left(t_{\mathrm{dec}}^{\Phi}\right)}{s_{T}\left(t_{\mathrm{dec}}^{\Phi}\right)} \simeq\left(\frac{\rho_{\Phi}\left(t_{\mathrm{dec}}^{\Phi}\right)}{\rho_{T}\left(t_{\mathrm{dec}}^{\Phi}\right)}\right)^{3 / 4} \simeq\left(\frac{\rho_{\Phi}\left(t_{\mathrm{dec}}^{\Phi}\right)}{\rho_{\Phi}\left(t_{\mathrm{dec}}^{T}\right)} \frac{\rho_{T}\left(t_{\mathrm{dec}}^{T}\right)}{\rho_{T}\left(t_{\mathrm{dec}}^{\Phi}\right)} \frac{\rho_{\Phi}\left(t_{\mathrm{dec}}^{T}\right)}{\rho_{T}\left(t_{\mathrm{dec}}^{T}\right)}\right)^{3 / 4} \\
& \simeq\left[\left(\frac{a\left(t_{\mathrm{dec}}^{T}\right)}{a\left(t_{\mathrm{dec}}^{\Phi}\right)}\right)^{3}\left(\frac{a\left(t_{\mathrm{dec}}^{\Phi}\right)}{a\left(t_{\mathrm{dec}}^{T}\right)}\right)^{4}\right]^{3 / 4} \simeq\left(\frac{a\left(t_{\mathrm{dec}}^{\Phi}\right)}{a\left(t_{\mathrm{dec}}^{T}\right)}\right)^{3 / 4} \simeq\left(\frac{t_{\mathrm{dec}}^{\Phi}}{t_{\mathrm{dec}}^{T}}\right)^{1 / 2} \\
& \simeq\left(\frac{\Gamma_{\mathrm{tot}}^{T}}{\Gamma_{\mathrm{tot}}^{\Phi}}\right)^{1 / 2},
\end{aligned}
$$

which is much larger than unity under our assumption.

In this way, even if a lot of gravitino is generated at the modulus decay, huge entropy injection dilutes the gravitino and LSP abundance. Although we assume that $\Phi$ gravitationally couples to the matter fields and decays into them after the decay of modulus field until now, we expect that proposed dilution mechanism is applied in the general class of models. If there is a sizable kinetic mixing between $T$ and $\Phi$, the lightest scalar field which is the linear combination of $T$ and $\Phi$ would decays into the gravitino(s) after diagonalizing them. Note that, supersymmetric stabilization condition, $D_{\Phi} W=0$, is also discussed in the gravitino production from the inflaton decay [47]. In the next section, we demonstrate the above analysis within the framework of type II string theory.

\footnotetext{
${ }^{5}$ Note that the decay temperatures of real and imaginary parts of modulus are also the same as shown in eq. (2.9).
} 


\section{The dilution mechanism in effective action of type II string theory}

To make the analysis concrete, we show the dilution mechanism based on the effective action of type II string theory, e.g., type IIB string theory. As pointed out in ref. [30], we have often assumed that all the complex structure moduli and axion-dilaton are stabilized at the compactification scale by the three-form fluxes. However, the above statement depends on the ansatz of three-form fluxes. In this section, we assume that one of the complex structure moduli remains massless at the perturbative level in the flux compactification. In this case, this massless moduli $(U)$ appears through the non-perturbative effects in the prepotential (see for more details, e.g., ref. [48]),

$$
F=F_{\text {pert }}-\frac{n}{(2 \pi i)^{3}} e^{2 \pi i U},
$$

where $F_{\text {pert }}$ is the perturbative prepotential, and $n$ is the instanton number associated with the relevant cycle. Below the mass scales of other stabilized complex structure moduli and axion-dilaton, the Kähler potential in eq. (2.1) reduces to be

$$
\begin{aligned}
K= & -3 \ln (-i(T-\bar{T})) \\
& -\ln \left[U_{\mathcal{I}} \beta+\gamma+\frac{n}{2 \pi^{2}} U_{\mathcal{I}} e^{-2 \pi U_{\mathcal{I}}} \cos \left(2 \pi U_{\mathcal{R}}\right)+\frac{n}{2 \pi^{3}} e^{-2 \pi U_{\mathcal{I}}} \cos \left(2 \pi U_{\mathcal{R}}\right)\right],
\end{aligned}
$$

where $\beta$ and $\gamma$ are the positive real constant determined by the vacuum expectation values of other stabilized moduli fields. Here, we consider the vanishing self-intersection number for $U=U_{\mathcal{R}}+i U_{\mathcal{I}}$, for simplicity. It is straightforward to extend our following discussion to the case of nonvanishing self-intersection number of $U$.

Let us further assume that the superpotential does not depend on the remaining complex structure modulus $U$. This situation is ensured by choosing the $U$-independent threeform fluxes in eq. (2.2) and assuming that the threshold correction to the gauge coupling in the hidden sector is independent of $U[49,50]$. In terms of the effective superpotential described in eq. (2.3) and Kähler potential in eq. (4.2), the $F$-term scalar potential on the basis of $4 \mathrm{D} \mathcal{N}=1$ supergravity is given by

$$
V=e^{K}\left(K^{I \bar{J}} D_{I} W D_{\bar{J}} \bar{W}-3|W|^{2}\right),
$$

where $D_{I} W=W_{I}+K_{I} W, K_{I}=\partial K / \partial \Phi^{I}, W_{I}=\partial W / \partial \Phi^{I}$ with $\Phi^{I}=T, U$, and $K^{I \bar{J}}$ is the inverse of the Kähler metric $K_{I \bar{J}}$.

Although the stabilization condition of Kähler modulus is the same as that of KKLT moduli stabilization, $D_{T} W=0$, the complex structure modulus can be stabilized at the minimum satisfying

$$
\begin{aligned}
& K_{U_{\mathcal{I}}}=-\frac{\beta-\frac{n}{2 \pi^{2}} e^{-2 \pi U_{\mathcal{I}}} \cos \left(2 \pi U_{\mathcal{R}}\right)-\frac{n}{\pi} U_{\mathcal{I}} e^{-2 \pi U_{\mathcal{I}}} \cos \left(2 \pi U_{\mathcal{R}}\right)}{U_{\mathcal{I}} \beta+\gamma+\frac{n}{2 \pi^{2}} U_{\mathcal{I}} e^{-2 \pi U_{\mathcal{I}}} \cos \left(2 \pi U_{\mathcal{R}}\right)+\frac{n}{2 \pi^{3}} e^{-2 \pi U_{\mathcal{I}}} \cos \left(2 \pi U_{\mathcal{R}}\right)}=0, \\
& K_{U_{\mathcal{R}}}=\frac{\frac{n}{\pi} U_{\mathcal{I}} e^{-2 \pi U_{\mathcal{I}}} \sin \left(2 \pi U_{\mathcal{R}}\right)+\frac{n}{\pi^{2}} e^{-2 \pi U_{\mathcal{I}}} \sin \left(2 \pi U_{\mathcal{R}}\right)}{U_{\mathcal{I}} \beta+\gamma+\frac{n}{2 \pi^{2}} U_{\mathcal{I}} e^{-2 \pi U_{\mathcal{I}}} \cos \left(2 \pi U_{\mathcal{R}}\right)+\frac{n}{2 \pi^{3}} e^{-2 \pi U_{\mathcal{I}}} \cos \left(2 \pi U_{\mathcal{R}}\right)}=0,
\end{aligned}
$$


which are solved as

$$
\begin{aligned}
\left\langle U_{\mathcal{R}}\right\rangle & =0, \\
\beta-\frac{n}{2 \pi^{2}} e^{-2 \pi\left\langle U_{\mathcal{I}}\right\rangle}-\frac{n}{\pi}\left\langle U_{\mathcal{I}}\right\rangle e^{-2 \pi\left\langle U_{\mathcal{I}}\right\rangle} & =0 .
\end{aligned}
$$

With the parameters $\beta=1$ and $n=1000$, the vacuum expectation value of $U_{\mathcal{I}}$ becomes

$$
\left\langle U_{\mathcal{I}}\right\rangle \simeq 0.93
$$

in the string unit. Such numerical values of parameters and moduli vacuum expectation values are ensured as follows. As shown in refs. [51, 52], the large instanton number often appears in a certain CY manifold. It thus allows us to treat the small vacuum expectation values of complex structure modulus compared with the string length, since we treat the quantum-corrected prepotential. Note that Kähler and complex structure moduli are independently stabilized at the supersymmetric minimum, $D_{T} W=D_{U} W=0$.

However, the obtained supersymmetric minimum gives rise to a negative energy density of the scalar potential, i.e., AdS vacuum. In order to uplift the vacuum to dS vacuum, there are several uplifting scenario such as F-term uplifting [35-38], and explicit SUSY-breaking as an existence of anti D-brane [11]. Let us analyze the moduli masses and SUSY-breaking sector for each individual case.

\section{1 $\quad F$-term uplifting}

The SUSY-breaking sector living on hidden D-brane enables us to uplift the AdS vacuum to the dS vacuum with tiny cosmological constant [35-38]. In this section, we study three types of moduli stabilization with F-term uplifting scenario by the SUSY breaking such as the Intriligator-Seiberg-Shih scenario [53].

\subsubsection{Model 1}

First of all, we consider the following Kähler and superpotential on hidden sector in addition to the moduli Kähler potential in eq. (4.2) and superpotential in eq. (2.3),

$$
\begin{aligned}
& \Delta K=Z(T-\bar{T})|X|^{2}-Z^{(1)}(T-\bar{T}) \frac{|X|^{4}}{\Lambda^{2}}, \\
& \Delta W=\mu X,
\end{aligned}
$$

where $\mu$ is the real constant determined by the heavy moduli fields, and the four-point coupling of the SUSY-breaking multiplet $X$ in the Kähler potential appears after integrating out the heavy mode with mass $\Lambda$. We now assume that the Kähler metric of SUSY-breaking multiplet $X$ is independent of $U$, otherwise $U$ obtains the $F$-term. We come back to the case of $U$-dependent uplifting scenario in appendix A. From the Kähler potential (4.2) and superpotential (2.3) including the SUSY-breaking sector (4.7) at the supersymmetric minimum of moduli fields, $D_{T} W=D_{U} W=0$, the extremal condition of SUSY-breaking field $X$,

$$
\frac{\partial V}{\partial \bar{X}} \simeq \partial_{\bar{X}}\left[e^{K}\left(K^{X \bar{X}}\left|D_{X} W\right|^{2}-3|W|^{2}\right)\right] \simeq e^{K}\left(\partial_{\bar{X}}\left(K^{X \bar{X}}\right) \mu^{2}-2 \mu w_{0}\right) \simeq 0
$$


leads to the minimum of $X$,

$$
\langle X\rangle \simeq \frac{Z(T-\bar{T})^{2} w_{0}}{2 Z^{(1)}(T-\bar{T}) \mu} \Lambda^{2}
$$

which is much smaller than unity under $\Lambda \ll 1$. At this minimum, the tiny cosmological constant can be realized by properly choosing $\mu$ and $w_{0}$ as

$$
\langle V\rangle \simeq e^{K}\left[K^{X \bar{X}}\left|D_{X} W\right|^{2}-3|W|^{2}\right] \simeq e^{K}\left(\frac{\mu^{2}}{Z(T-\bar{T})}-3 w_{0}^{2}\right) \simeq 0 .
$$

However, the inclusion of SUSY-breaking sector violates the extremal conditions for the moduli fields. To find the true minimum of moduli fields $(T, U)$, we evaluate the deviations from the supersymmetric minimum, $\delta T=T-T_{\text {ref }}$ and $\delta U=U-U_{\text {ref }}$ where $T_{\text {ref }}$ and $U_{\text {ref }}$ are the reference points satisfying the supersymmetric conditions, $\left.D_{T} W\right|_{\text {ref }}=\left.D_{U} W\right|_{\text {ref }}=0$. The true minimum of SUSY-breaking field $X$ is also found by evaluating the deviation from the reference point in eq. (4.9), $\delta X=X-\langle X\rangle$. First of all, let us take a closer look at $\delta U$. As far as the SUSY-breaking field does not couple to $U$, the extremal condition of $U$, $\partial V / \partial U=0$, is always satisfied under the supersymmetric condition, $K_{U}=0$, i.e., $\delta U=0$.

Next, we evaluate the variations, $\delta T$ and $\delta X$, in terms of

$$
\begin{aligned}
& D_{T} W=\left.W_{T T}\right|_{\mathrm{ref}} \delta T+\left.\left(K_{T} W_{T}\right)\right|_{\mathrm{ref}} \delta T+\left.\left(K_{T} W_{X}\right)\right|_{\mathrm{ref}} \delta X+\left.\left(K_{T \bar{T}} W\right)\right|_{\mathrm{ref}}(\delta \bar{T}-\delta T), \\
& \left.D_{X} W \simeq W_{X}\right|_{\mathrm{ref}}+\left.\left(K_{X} W_{T}\right)\right|_{\mathrm{ref}} \delta T+\left.\left(K_{X \bar{X}} W\right)\right|_{\mathrm{ref}}(\delta \bar{X}-\delta X)
\end{aligned}
$$

where we take the limit $\langle X\rangle \ll 1$. From the scalar potential at quadratic order of $\delta T$ and $\delta X$,

$$
\begin{aligned}
V= & \left.V\right|_{\mathrm{ref}}+\left.V_{I}\right|_{\mathrm{ref}} \delta \phi^{I}+\left.V_{\bar{I}}\right|_{\mathrm{ref}} \delta \bar{\phi}^{I} \\
& +\left.\frac{1}{2} V_{I J}\right|_{\mathrm{ref}} \delta \phi^{I} \delta \phi^{J}+\left.V_{I \bar{J}}\right|_{\mathrm{ref}} \delta \phi^{I} \delta \bar{\phi}^{J}+\left.\frac{1}{2} V_{\bar{I} \bar{J}}\right|_{\mathrm{ref}} \delta \bar{\phi}^{I} \delta \bar{\phi}^{J}
\end{aligned}
$$

with $\left.V_{I}\right|_{\text {ref }}=\left.\partial_{I} V\right|_{\text {ref }}$ and $\left.V_{I J}\right|_{\text {ref }}=\left.\partial_{I} \partial_{J} V\right|_{\text {ref }}$ being the first and second derivatives with respect to the fields, $\phi^{I}=T, X$, at their reference points, we obtain the variations of $T$ and $X$

$$
\begin{aligned}
& \delta T \simeq \frac{9|W|^{2}}{2 T_{\mathcal{I}} K^{T \bar{T}}\left|W_{T T}\right|^{2}} \simeq \frac{3 i}{2 T_{\mathcal{I}} a^{2}} \\
& \delta X \simeq \frac{\sqrt{3} Z(T-\bar{T})^{3 / 2}}{6 Z^{(1)}(T-\bar{T})} \Lambda^{2}
\end{aligned}
$$

in which our analysis is justified due to the following equality:

$$
\left|V_{I}\right|_{\mathrm{ref}} \delta \phi^{I}+\left.\left.V_{\bar{I}}\right|_{\mathrm{ref}} \delta \bar{\phi}^{I}|\gg| \frac{1}{2} V_{I J}\right|_{\mathrm{ref}} \delta \phi^{I} \delta \phi^{J}+\left.V_{I \bar{J}}\right|_{\mathrm{ref}} \delta \phi^{I} \delta \bar{\phi}^{J}+\left.\frac{1}{2} V_{\bar{I} \bar{J}}\right|_{\mathrm{ref}} \delta \bar{\phi}^{I} \delta \bar{\phi}^{J} \mid .
$$

Since there is no kinetic mixing between $U$ and $T$, the mass squared of canonically normalized moduli fields are evaluated at the obtained minimum, $T=T_{\text {ref }}+\delta T, U=U_{\text {ref }}$, 
and $X=X_{\text {ref }}+\delta X$,

$$
\begin{aligned}
& m_{U_{\mathcal{R}}}^{2}=\frac{V_{U_{\mathcal{R}} U_{\mathcal{R}}}}{2 K_{U \bar{U}}}=\frac{1}{4}\left(\frac{K_{U_{\mathcal{R}} U_{\mathcal{R}}}}{K_{U \bar{U}}}\right)^{2} m_{3 / 2}^{2}=(2 \pi)^{2}\left(U_{\mathcal{I}}+\frac{1}{\pi}\right)^{2} m_{3 / 2}^{2}, \\
& m_{U_{\mathcal{I}}}^{2}=\frac{V_{U_{\mathcal{I}} U_{\mathcal{I}}}}{2 K_{U \bar{U}}}=\frac{1}{4}\left(\frac{K_{U_{\mathcal{I}} U_{\mathcal{I}}}}{K_{U \bar{U}}}\right)^{2} m_{3 / 2}^{2}=(2 \pi)^{2} U_{\mathcal{I}}^{2} m_{3 / 2}^{2}, \\
& m_{T_{\mathcal{R}}}^{2}=\frac{V_{T_{\mathcal{R}} T_{\mathcal{R}}}}{2 K_{T \bar{T}}}=\frac{3 a^{2}}{2 K_{T \bar{T}}} m_{3 / 2}^{2} \simeq\left(2 a T_{\mathcal{I}}\right)^{2} m_{3 / 2}^{2}, \\
& m_{T_{\mathcal{I}}}^{2} \simeq m_{T_{\mathcal{R}}}^{2}
\end{aligned}
$$

whereas that of SUSY-breaking field is given by $m_{X_{\mathcal{R}}}^{2} \simeq m_{X_{\mathcal{I}}}^{2} \simeq \frac{6 Z^{(1)}(T-\bar{T})}{Z(T-\bar{T})^{2} \Lambda^{2}} m_{3 / 2}^{2}$, which can be taken larger than the moduli masses. Therefore, moduli decay into the dynamical SUSYbreaking sector is neglected. With $U_{\mathcal{I}}<1$ and $a T_{\mathcal{I}} \sim 4 \pi^{2}$, the mass squared of complex structure modulus is typically smaller than that of Kähler modulus. Furthermore, the corresponding $F$-terms are given by

$$
\begin{aligned}
\left\langle\frac{F^{T}}{T-\bar{T}}\right\rangle & \simeq \frac{3}{2 a T_{\mathcal{I}}} m_{3 / 2} \simeq 3 \frac{m_{3 / 2}^{2}}{m_{T_{\mathcal{I}}}}, \\
\left\langle F^{U}\right\rangle & =0 \\
\left\langle F^{X}\right\rangle & \simeq-\sqrt{\frac{3}{Z(T-\bar{T})}} m_{3 / 2} .
\end{aligned}
$$

As a result, the complex structure modulus lighter than Kähler modulus does not violate the SUSY at the vacuum. When the SUSY-breaking field has $U$-dependent Kähler metric in eq. (4.7), the complex structure modulus induces the SUSY-breaking as studied in appendix A.

\subsubsection{Model 2}

Next, we comment on another Kähler moduli stabilization, in contrast to the KKLT-type. The detail of following procedure is the same as previous one in section 4.1.1. ${ }^{6}$ When the Kähler modulus is stabilized at the racetrack minimum in the superpontential,

$$
W=w_{0}+B e^{i b T}-C e^{i c T}
$$

where two non-perturbative effects are generated associated with the cycle $T$ through the hidden extra D7-branes with $b \sim c \sim 4 \pi^{2}$, the supersymmetric minimum of modulus field is found as

$$
T_{\mathrm{ref}} \sim \frac{i}{b-c} \ln \left(\frac{b B}{c C}\right) .
$$

\footnotetext{
${ }^{6}$ It is summarized in e.g., refs. [36, 38].
} 
By combing the SUSY-breaking sector in eq. (4.7) and racetrack scenario, we find that the deviations from the reference points given in eqs. (4.9) and (4.18) are

$$
\begin{gathered}
\delta T \simeq i \frac{9|W|^{2}}{2 T_{\mathcal{I}} K^{T \bar{T}}\left|W_{T T}\right|^{2}}, \\
\delta X \simeq \frac{\sqrt{3} Z(T-\bar{T})^{3 / 2}}{6 Z^{(1)}(T-\bar{T})} \Lambda^{2},
\end{gathered}
$$

at which the mass squared of Kähler modulus and the corresponding $F$-term become

$$
\begin{aligned}
m_{T_{\mathcal{I}}}^{2} & \simeq m_{T_{\mathcal{R}}}^{2} \simeq \frac{16 Z(T-\bar{T})}{3}(b c)^{2} T_{\mathcal{I}}^{4} m_{3 / 2}^{2}\left[\left(1-\frac{b}{c}\right) \frac{B e^{-b T_{\mathcal{I}}}}{\mu}\right]^{2}, \\
\left\langle\frac{F^{T}}{T-\bar{T}}\right\rangle & \simeq \frac{3\left(b B e^{-b T_{\mathcal{I}}}-c C e^{-c T_{\mathcal{I}}}\right)}{2 T_{\mathcal{I}}\left(-b^{2} B e^{-b T_{\mathcal{I}}}+c^{2} C e^{-c T_{\mathcal{I}}}\right)} m_{3 / 2} \simeq 3 \frac{m_{3 / 2}^{2}}{m_{T_{\mathcal{I}}}}
\end{aligned}
$$

The mass squared and $F$-term of complex structure modulus and SUSY-breaking field are the same as in section 4.1.1. Thus, in contrast to the single non-perturbative effect for $T$, the Kähler modulus is much heavier than the light complex structure modulus. According to it, the $F$-term of Kähler modulus is more suppressed than the case of single non-perturbative effect.

\subsubsection{Model 3}

Finally, we discuss another $F$-term uplifting scenario combined with the racetrack superpotential in eq. (4.17). The Kähler potential and superpotential of hidden sector are described by

$$
\begin{aligned}
& \Delta K=Z(T-\bar{T})|X|^{2}-Z^{(1)}(T-\bar{T}) \frac{|X|^{4}}{\Lambda^{2}} \\
& \Delta W=D e^{i d T} X
\end{aligned}
$$

where the above superpotential can be generated by the gaugino condensation and D-brane instanton effects. In a similar way to the discussion in sections 4.1.1 and 4.1.2, the reference points of moduli fields and SUSY-breaking fields are the same as one obtained above. However, the superpotential (4.21) leads to the following deviations from the reference points,

$$
\begin{aligned}
& \delta T \simeq i \frac{3 d|W|^{2}}{K^{T \bar{T}}\left|W_{T T}\right|^{2}} \simeq i \frac{9 d|W|^{2}}{4 T_{\mathcal{I}}^{2}\left(-b^{2} B e^{-b T_{I}}+c^{2} C e^{-c T_{I}}\right)^{2}}, \\
& \delta X \simeq \frac{\sqrt{3} Z(T-\bar{T})^{3 / 2}}{6 Z^{(1)}(T-\bar{T})} \Lambda^{2}\left[1+\frac{\sqrt{3} d^{2} W}{2\left(-b^{2} B e^{-b T_{I}}+c^{2} C e^{\left.-c T_{I}\right)}\right.}\right]
\end{aligned}
$$

at which the mass squared of Kähler modulus and the corresponding $F$-term become

$$
\begin{aligned}
m_{T_{\mathcal{I}}}^{2} & \simeq m_{T_{\mathcal{R}}}^{2} \simeq \frac{16 Z(T-\bar{T})}{3}(b c)^{2} T_{\mathcal{I}}^{4} m_{3 / 2}^{2}\left[\frac{B}{D}\left(1-\frac{b}{c}\right) e^{-(b-d) T_{\mathcal{I}}}\right]^{2}, \\
\left\langle\frac{F^{T}}{T-\bar{T}}\right\rangle & \simeq \frac{3 d}{2 T_{\mathcal{I}}} \frac{m_{3 / 2}^{2}}{m_{T_{\mathcal{I}}}}
\end{aligned}
$$


The mass squared and $F$-term of complex structure modulus and SUSY-breaking field are the same as in section 4.1.1, whereas the mass and $F$-term of Kähler modulus are different from the previous setups in sections 4.1.2 and 4.1.3. With the parameter $d \simeq 4 \pi^{2}$, it turns out that only the $F$-term is taken to be larger than that in section 4.1.2.

\subsection{Uplifting with anti D-brane}

In this section, we show another uplifting scenario as an existence of anti D-brane [11] located at a certain warped throat. Tiny cosmological constant is realized from the positive vacuum energy originating from the anti D-branes, although these anti D-branes induce the explicit SUSY-breaking in contrast to the spontaneous SUSY-breaking in section 4.1. As proposed in refs. $[1,2]$, the uplifting term is formulated in the $4 \mathrm{D}$ effective supergravity,

$$
\mathcal{L}_{\text {up }}=-\int d^{4} \theta|C|^{4} \theta^{2} \bar{\theta}^{2} \mathcal{P}
$$

where $\mathcal{P}$ denotes the unknown function of moduli fields. Then, we obtain the uplifting scalar potential is given by

$$
V_{\text {up }}=e^{2 K / 3} \mathcal{P}
$$

When the $\mathcal{P}$ is independent of $U$, the complex structure modulus $U$ cannot be deviated from the supersymmetric minimum, $K_{U}=0$. However, the mass squared of $U$ receives the contribution from the uplifting sector,

$$
\begin{aligned}
& m_{U_{\mathcal{R}}}^{2}=(2 \pi)^{2}\left(U_{\mathcal{I}}+\frac{1}{\pi}\right)^{2} m_{3 / 2}^{2}+4 \pi\left(U_{\mathcal{I}}+\frac{1}{\pi}\right) m_{3 / 2}^{2}, \\
& m_{U_{\mathcal{I}}}^{2}=(2 \pi)^{2} U_{\mathcal{I}}^{2} m_{3 / 2}^{2}+4 \pi U_{\mathcal{I}} m_{3 / 2}^{2} .
\end{aligned}
$$

Thus, the SUSY is only broken by the Kähler moduli. Along the same step outlined in section 4.1, the variation of $T$ and corresponding $F$-term are evaluated as

$$
\begin{aligned}
\delta T & \simeq \frac{5 i}{2 a^{2} T_{\mathcal{I}}}\left[1-\frac{2 i T_{\mathcal{I}}}{5} \partial_{T} \ln (\mathcal{P})\right], \\
\left\langle\frac{F^{T}}{T-\bar{T}}\right\rangle & \simeq \frac{1}{a T_{\mathcal{I}}} m_{3 / 2}\left(\frac{5}{2}-i T_{\mathcal{I}} \partial_{T} \ln (\mathcal{P})\right),
\end{aligned}
$$

at which the mass squared of $T$ is approximately the same in eq. (3.4). Throughout the discussion in sections 4.1 and 4.2 , the complex structure modulus does not induce the SUSY-breaking at the uplifted minimum, if the uplifting sector is independent of the complex structure modulus. Thus, it can play a role of diluting the gravitino abundance as discussed in section 3. In the next section, we focus on the moduli dynamics after the inflationary era.

\subsection{The dilution mechanism}

In this section, we repeat the dilution mechanism in section 3 by identifying $\Phi$ as the lightest complex structure modulus $U$. In a way similar to the step in section 3 , we define 
the true minimum of moduli fields as $T_{0}$ in eq. (2.4) for the Kähler modulus and $U_{0}$ in eq. (4.5) for the lightest complex structure modulus, respectively. When the Hubble scale $H$ is comparable to the mass scale of moduli fields, $m_{T}$ and $m_{U}$, moduli fields roll down to the true minimum and dominate the energy density of the Universe as mentioned in section 3. The scalar potential of our interest is

$$
V_{\mathrm{osc}}=\frac{H^{2}(t)}{2}\left|T-T_{1}\right|^{2}+\frac{m_{T}^{2}}{2}\left|T-T_{0}\right|^{2}+\frac{H^{2}(t)}{2}\left|U-U_{1}\right|^{2}+\frac{m_{U}^{2}}{2}\left|U-U_{0}\right|^{2},
$$

where $U_{1}$ and $T_{1}$ denote the minimum induced by the Hubble parameter $H(t)$. Here, we employ the canonically normalized moduli fields by the same notation $U$ and $T$. In our model, the lightest complex structure modulus $U$ is lighter than the Kähler modulus $T$, i.e., $m_{U}<m_{T}$. Thus, $U$ oscillates subsequent to the oscillation of $T$, i.e., $t_{\mathrm{osc}}^{U}>t_{\mathrm{osc}}^{T}$, where the oscillating time of moduli fields $U$ and $T$ are defined as $t_{\mathrm{osc}}^{U}$ and $t_{\mathrm{osc}}^{T}$, respectively. As shown in section 3, when we assume that the initial displacement of moduli fields as $\Delta T \simeq \Delta U=\mathcal{O}\left(M_{\mathrm{Pl}}\right)$, the oscillating energy densities of $U$ and $T$ at the time $t_{\mathrm{osc}}^{U} \simeq 1 / m_{U}$ are almost the same as each other,

$$
\frac{1}{2} m_{T}^{2} T\left(t_{\mathrm{osc}}^{U}\right)^{2} \simeq \frac{1}{2} m_{U}^{2}(\Delta U)^{2}
$$

It implies that two moduli fields dominate the energy densities of Universe at the time $t_{\mathrm{osc}}^{U}$.

Next, let us move onto the reheating process of both moduli fields. As analyzed in eq. (2.9), the Kähler modulus dominantly decays into the gauge boson pairs, whereas the complex structure modulus does not appear in the gauge kinetic function at the tree-level. At the one-loop level, the gauge kinetic function has $U$-dependence through the threshold correction to the gauge coupling in the visible sector. We find that the one-loop gauge kinetic functions in the visible sector are brought into the following form,

$$
f_{a}=k T+\frac{1}{16 \pi^{2}} \Delta_{a}(U)
$$

where the threshold corrections $\Delta_{a}(U)$ are only calculated in the case of toroidal compactification $[49,50]$. In this case, the authors of refs. $[49,50]$ show that $\Delta_{a}(U)$ is written in terms of the Dedekind eta function and beta-function coefficient for the charged strings in the $\mathcal{N}=2$ sector. Thus, the decay width from $U$ into gauge boson pairs is suppressed by that of Kähler modulus. It is expected that the other possible decay channels are arisen from the kinetic terms of matter fields in the Kähler potential and Yukawa couplings in the superpotential. However, the axion associated with the lightest complex structure modulus $U, U_{\mathcal{R}}$, only appears in the kinetic terms of matter fields and Yukawa couplings through the non-perturbative effects due to the invariance of (discrete) shift symmetry. In this way, we assume that the lifetime of axion $U_{\mathcal{R}}$ becomes longer compared with $U_{\mathcal{I}}$ which appears in the tree-level Kähler and superpotentials, for simplicity. (See for example, ref. [54].) On the other hand, the decay temperatures of real and imaginary parts of $T$ are the same as shown in eq. (2.9). As a result, the sizable decay channels of $U_{\mathcal{R}}$ are mainly determined 
through the gauge kinetic function (4.30) and the total decay width of $U_{\mathcal{R}}$ is estimated as

$$
\begin{aligned}
\Gamma_{\text {tot }}^{U_{\mathcal{R}}} & =\sum_{a=1}^{3} \frac{N^{a}}{128 \pi}\left(\frac{\partial_{U} \Delta_{a}(U)}{16 \pi^{2}}\right)^{2}\left(\frac{g_{a}^{2}}{\sqrt{\left\langle K_{U \bar{U}}\right\rangle}}\right)^{2} \frac{m_{U_{\mathcal{R}}}^{3}}{M_{\mathrm{Pl}}^{2}} \\
& \simeq 2.3 \times 10^{-23}\left(\frac{\langle\Delta(U)\rangle}{20}\right)^{2}\left\langle K^{U \bar{U}}\right\rangle\left(\frac{m_{U_{\mathcal{R}}}}{10^{6} \mathrm{GeV}}\right)^{3} \mathrm{GeV},
\end{aligned}
$$

with $\Delta(U)=\sum_{a} \partial_{U} \Delta_{a}(U), N^{a}=\{8,3,1\}$ and $\left(g_{a}\right)^{2} \simeq 0.53$, which gives rise to a radiationdominated Universe with a reheating temperature,

$$
\begin{aligned}
T_{\mathrm{reh}}^{U_{\mathcal{R}}} & \simeq\left(\frac{\pi^{2} g_{*}\left(T_{\mathrm{reh}}^{U_{\mathcal{R}}}\right)}{90}\right)^{-1 / 4} \sqrt{M_{\mathrm{Pl}} \Gamma_{\mathrm{tot}}^{U_{\mathcal{R}}}} \\
& \simeq 7.2\left(\frac{\langle\Delta(U)\rangle}{20}\right)\left\langle K^{U \bar{U}}\right\rangle^{1 / 2}\left(\frac{m_{U_{\mathcal{R}}}}{10^{6} \mathrm{GeV}}\right)^{3 / 2} \mathrm{MeV}
\end{aligned}
$$

with $g_{*}\left(T_{\text {reh }}^{U_{\mathcal{R}}}\right) \simeq 10.75$ being the effective number of degrees of freedom in the radiationdominated Universe.

It is argued that the lightest complex structure modulus $U_{\mathcal{R}}$ dominates the energy density of the Universe. Furthermore, this lightest modulus does not decay into the gravitino(s) due to the vanishing $F$-term of $U$. At the decay time of $U_{\mathcal{R}}, t_{\mathrm{dec}}^{U_{\mathcal{R}}}$, the produced entropy dilutes the gravitino (and LSP) yield given through the decay of $T$ at the time $t_{\mathrm{dec}}^{T}$. The dilution factor defined by the ratio between the entropy density of $U_{\mathcal{R}}\left(s_{U_{\mathcal{R}}}\right)$, and that of $T\left(s_{T}\right)$, at the time $t_{\mathrm{dec}}^{U_{\mathcal{R}}}$ is given by

$$
\begin{aligned}
\Delta_{S} & \simeq \frac{s_{U_{\mathcal{R}}}\left(t_{\mathrm{dec}}^{U_{\mathcal{R}}}\right)}{s_{T}\left(t_{\mathrm{dec}}^{U_{\mathcal{R}}}\right)} \simeq\left(\frac{\Gamma_{\text {tot }}^{T}}{\Gamma_{\text {tot }}^{U_{\mathcal{R}}}}\right)^{1 / 2} \\
& \simeq 223\left(\frac{\left\langle K^{T \bar{T}}\right\rangle}{\left\langle K^{U \bar{U}}\right\rangle}\right)^{1 / 2}\left(\frac{m_{T_{\mathcal{R}}}}{m_{U_{\mathcal{R}}}}\right)^{3 / 2}\left(\frac{|k|}{\langle\Delta(U)\rangle}\right),
\end{aligned}
$$

from which the mass hierarchy between $m_{T_{\mathcal{R}}}$ and $m_{U_{\mathcal{R}}}$ leads the enhancement of dilution factor. In sections 4.1 and 4.2 , we demonstrate the relation $m_{T_{\mathcal{R}}} \gg m_{U_{\mathcal{R}}}$ in the KKLT-and racetrack-type moduli stabilizations, respectively.

For the gravitino mass of $m_{3 / 2} \simeq \mathcal{O}(30) \mathrm{TeV}$, the BBN severely constrains the branching ratio $B_{3 / 2}$ in the modulus decay. However, the large dilution factor $\Delta_{S}$ reduces the gravitino yield at the time $t_{\mathrm{dec}}^{U_{\mathcal{R}}}$ to an acceptable level,

$$
\begin{aligned}
Y_{3 / 2}\left(t_{\mathrm{dec}}^{U_{\mathcal{R}}}\right)=\frac{n_{3 / 2}}{s_{U_{\mathcal{R}}}\left(t_{\mathrm{dec}}^{U_{\mathcal{R}}}\right)}=\frac{Y_{3 / 2}\left(t_{\mathrm{dec}}^{T}\right)}{\Delta_{S}} \simeq & B_{3 / 2} \frac{3 T_{\mathrm{reh}}^{U_{\mathcal{R}}}}{2 m_{T}} \\
\simeq & 4.8 \times 10^{-13}\left(\frac{B_{3 / 2}}{0.01}\right)\left(\frac{\langle\Delta(U)\rangle}{50}\right)\left\langle K^{U \bar{U}}\right\rangle^{1 / 2} \\
& \cdot\left(\frac{m_{U_{\mathcal{R}}}}{2 \times 10^{5} \mathrm{GeV}}\right)^{3 / 2}\left(\frac{5 \times 10^{7} \mathrm{GeV}}{m_{T}}\right)
\end{aligned}
$$


Even if the gravitino is much larger than $\mathcal{O}(30) \mathrm{TeV}$, the LSP yield produced by gravitino decay is also diluted by the entropy production of $U_{\mathcal{R}}$,

$$
Y_{\mathrm{LSP}}\left(t_{\mathrm{dec}}^{U_{\mathcal{R}}}\right)=\frac{Y_{\mathrm{LSP}}\left(t_{\mathrm{dec}}^{T}\right)}{\Delta_{S}}
$$

Since the large amount of gravitino is generated by the modulus decay in the high-scale SUSY-breaking scenario, the dark matter abundance is mainly dominated by the second term in eq. (2.15),

$$
\begin{aligned}
\Omega_{\mathrm{LSP}} h^{2} \simeq & m_{\mathrm{LSP}} Y_{\mathrm{LSP}}\left(t_{\mathrm{dec}}^{U_{\mathcal{R}}}\right) \frac{s_{\mathrm{now}}}{\rho_{\mathrm{cr}}} \\
\simeq & m_{\mathrm{LSP}} \sqrt{\frac{45}{8 \pi^{2} g_{*}\left(T_{\mathrm{reh}}^{U_{\mathcal{R}}}\right)}} \frac{1}{M_{\mathrm{Pl}} T_{3 / 2}\left\langle\sigma_{\mathrm{ann}} v\right\rangle} \frac{s_{\mathrm{now}}}{\rho_{\mathrm{cr}}}\left(\frac{\Gamma_{\mathrm{tot}}^{U_{\mathcal{R}}}}{\Gamma_{\mathrm{tot}}^{T}}\right)^{1 / 2} \\
\simeq & 0.3\left(\frac{80}{g_{*}\left(T_{3 / 2}\right)}\right)^{1 / 2}\left(\frac{10^{6} \mathrm{GeV}}{m_{3 / 2}}\right)^{3 / 2}\left(\frac{m_{\mathrm{LSP}}}{1 \mathrm{TeV}}\right)^{3}\left(\frac{10^{-3} \mathrm{GeV}^{-2}}{m_{\mathrm{LSP}}^{2}\left\langle\sigma_{\mathrm{ann}} v\right\rangle}\right)\left(\frac{m_{U_{\mathcal{R}}}}{m_{T_{\mathcal{R}}}}\right)^{3 / 2} \\
& \cdot\left(\frac{\langle\Delta(U)\rangle}{|k|}\right)\left(\frac{\left\langle K^{U \bar{U}}\right\rangle}{\left\langle K^{T \bar{T}}\right\rangle}\right)^{1 / 2},
\end{aligned}
$$

which is inversely proportional to the gravitino mass. Note that two moduli masses $\left(m_{U_{\mathcal{R}}}\right.$, $\left.m_{T_{\mathcal{R}}}\right)$ are both proportionally to the gravitino mass as discussed in sections 4.1 and 4.2. Thus, even if the gravitino mass is much heavier than $\mathcal{O}(30) \mathrm{TeV}$, the overabundance of the dark matter can be avoided in high-scale SUSY-breaking scenario. With the sample values of parameters achieved in the KKLT scenario,

$$
\langle\Delta(U)\rangle=10, \quad|k|=1, \quad\left\langle K^{U \bar{U}}\right\rangle \simeq\left\langle K^{T \bar{T}}\right\rangle \simeq 1, \quad m_{T_{\mathcal{R}}} \simeq 4 \pi^{2} m_{3 / 2}, \quad m_{U_{\mathcal{R}}} \simeq 2 \pi m_{3 / 2},
$$

we find that the mass squared of gravitino and LSP is constrained to account for the observed dark matter abundance as illustrated in figure 1, where the left (right) panel considers the Wino-like (Higgsino-like) neutralino, respectively. Although we now focus on two LSP scenarios within the mass range $100 \mathrm{GeV}<m_{\mathrm{LSP}}<2000 \mathrm{GeV}$ predicted by the mirage or anomaly mediations with gravitino mass $100 \mathrm{TeV}<m_{3 / 2}<1000 \mathrm{TeV}$, it is straightforward to extend our analysis to other dark matter scenarios with different SUSY-breaking scale.

As a result, in both the low-and high-scale SUSY breaking scenarios, the gravitino abundance is diluted by the huge entropy injection of the extra modulus field. In our scenario, the dilution mechanism is achieved by the existence of the lightest modulus field without breaking the SUSY. Although the kinetic mixing between $T$ and $U$ has the potential for causing the gravitino production, the $\mathcal{N}=2$ supersymmetric structure ensures the absence of kinetic mixing between moduli fields.

The dilution mechanism also has significant influences on the dark matter abundance of axion and the baryogenesis. Indeed, the entropy dilution allows us to treat the large decay constant of axion dark matter, compared with the usually considered axion dark 

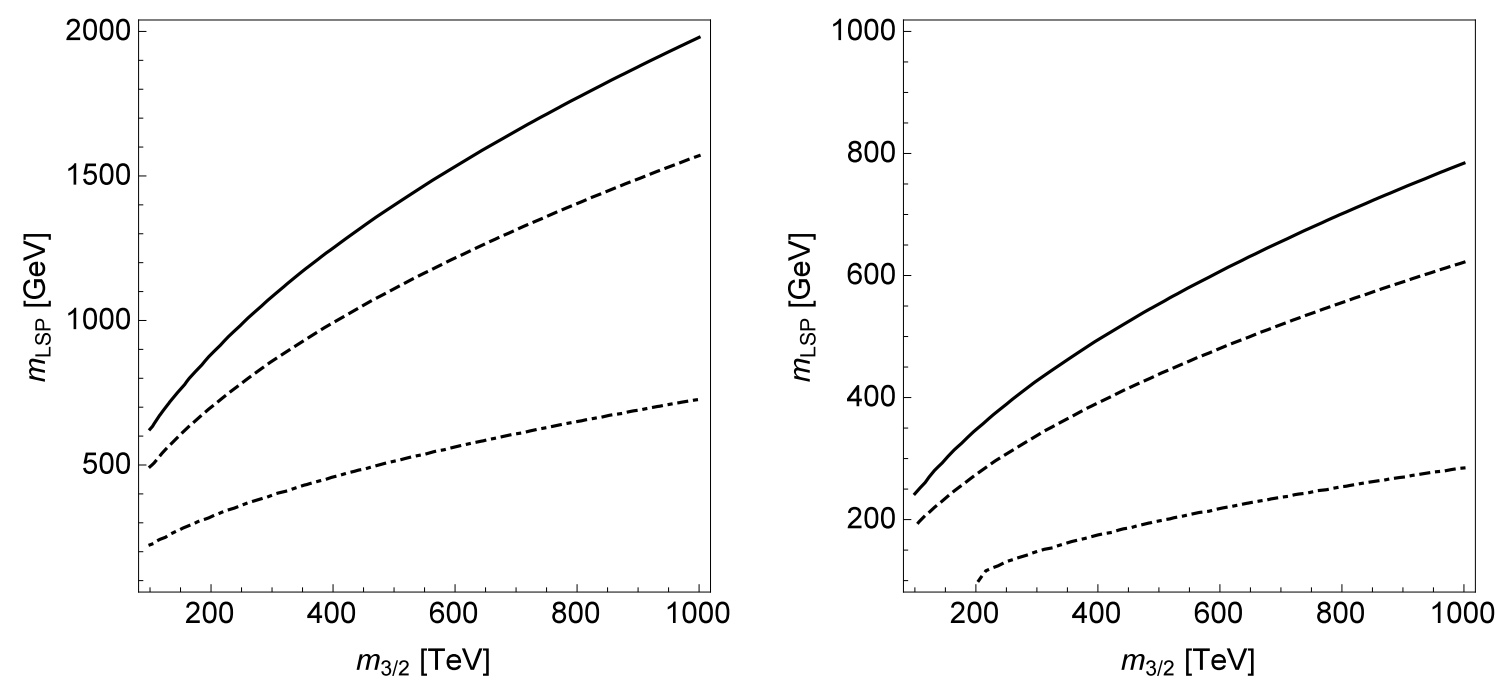

Figure 1. The dark matter abundance on the $\left(m_{3 / 2}, m_{\mathrm{LSP}}\right)$-plane, where LSP is identified as the Wino-like (Higgsino-like) neturalino in the left (right) panel. The black solid, dashed, and dotdashed curves correspond to $\Omega_{\mathrm{LSP}} h^{2}=0.2, \Omega_{\mathrm{LSP}} h^{2}=0.1$, and $\Omega_{\mathrm{LSP}} h^{2}=0.01$, respectively.

matter [55]. Since the string theory naturally predicts the large axion decay constant, the axion could then become a plausible candidate of the cold dark matter. On the other hand, the late entropy dilution may face a problem to explain the present baryon asymmetry. However, the large initial baryon asymmetry before the moduli oscillation would lead to the nonnegligible baryon asymmetry, through the Affleck-Dine mechanism [56, 57]. We leave the detailed study of the baryon asymmetry to the future work.

\section{Conclusion}

We have discussed the cosmological aspects of moduli and mirage mediations with an emphasis on the moduli-induced gravitino problem. Since the moduli fields gravitationally couples to the matter fields, they oscillate around their true minimum at a low temperature. The decay of moduli fields then generates the huge amount of gravitinos which can spoil the successful BBN in the low-scale SUSY-breaking scenario and cause the overabundance of LSP in the high-scale SUSY-breaking scenario, respectively. It is a common feature in the low-energy effective action originating from higher-dimensional theory, in particular, string theory. So far, there were several proposals to dilute the gravitinos via the thermal inflation [21, 22], Q-ball [23] and unstable domain-wall decays [24], or the introduction of the axion sector [25].

In this paper, we explore another dilution mechanism by taking into account the extra modulus field. In contrast to the KKLT-type moduli stabilization mechanism [11], the extra modulus field has been stabilized at the supersymmetric minimum, as far as the uplifting sector does not depend on the extra modulus field. Thus, such extra field does not decay into the gravitinos at its decay. Furthermore, in the KKLT-type moduli stabilization, we have shown that this extra modulus field dominates the energy density of the Universe after 
the time when the volume modulus field dominates. This situation has been achieved when the extra modulus field is lighter than the volume modulus and at the same time, the decay time of extra modulus is later than that of volume modulus. Within the framework of type IIB (IIA) string theory, the lightest complex structure (Kähler) modulus corresponds to this plausible candidate. Thus, the mirage mediation is desirable scenario not only from the aspects of fine-tuning problem, but also from the cosmological aspects.

\section{Acknowledgments}

The authors thank H. Abe for useful discussions. T.K. was supported in part by the Grant-in-Aid for Scientific Research No. 25400252 and No. 26247042 from the Ministry of Education, Culture, Sports, Science and Technology (MEXT) in Japan. H.O. was supported in part by a Grant-in-Aid for JSPS Fellows No. 26-7296.

\section{A Moduli-dependent uplifting scenario}

We comment on the case that the uplifting sector depends on lightest complex structure modulus $U$, in particular, the uplifting scenario with anti D-branes. As a consequence of anti D-branes, the uplifting potential is generically described by

$$
V_{\text {up }}=e^{2 K / 3} \mathcal{P}(T-\bar{T}, U-\bar{U}),
$$

where $\mathcal{P}$ is the function of moduli fields $T$ and $U$, in general. In contrast to the discussion in section 4.2, the minimum of the lightest complex structure modulus $U$ can be deviated from the supersymmetric minimum, $K_{U}=0$. Along the same step outlined in the section 4.1, the variations of $T$ and $U$ are evaluated in terms of

$$
\begin{aligned}
& D_{T} W=\left.W_{T T}\right|_{\mathrm{ref}} \delta T+\left.\left(K_{T} W_{T}\right)\right|_{\mathrm{ref}} \delta T+\left.\left(K_{T \bar{T}} W\right)\right|_{\mathrm{ref}}(\delta \bar{T}-\delta T) \\
& D_{U} W=\left.\left(K_{U U} W\right)\right|_{\mathrm{ref}} \delta U+\left.\left(K_{U \bar{U}} W\right)\right|_{\mathrm{ref}} \delta \bar{U} .
\end{aligned}
$$

At quadratic order of $\delta T$ and $\delta U$ in the scalar potential, we obtain the variations of $T$ and $U$

$$
\begin{aligned}
& \delta T \simeq \frac{5 i}{2 a^{2} T_{\mathcal{I}}}\left[1-\frac{2 i T_{\mathcal{I}}}{5} \partial_{T} \ln (\mathcal{P})\right]+\frac{4 \partial_{U} \ln (\mathcal{P})\left(\frac{\partial_{T} \partial_{U} P}{P}+\frac{i}{T_{\mathcal{I}}} \partial_{U} \ln (\mathcal{P})\right)}{a^{2}\left[\frac{K^{U \bar{U}}}{6}\left(K_{U_{\mathcal{I}} U_{\mathcal{I}}}\right)^{2}-4\left(\frac{2}{3} K_{U U}+\frac{\partial_{U} \partial_{U} P}{P}\right)\right]}, \\
& \delta U \simeq \frac{2 \partial_{U} \ln (\mathcal{P})}{\frac{K^{U \bar{U}}\left(K_{U_{\mathcal{I}} U_{\mathcal{I}}}\right)^{2}}{6}-4\left(\frac{2}{3} K_{U U}+\frac{\partial_{U} \partial_{U} P}{P}\right)} .
\end{aligned}
$$

Since there is no kinetic mixing between $U$ and $T$, the mass squared of canonically normalized moduli fields are evaluated at the obtained minimum, $T=T_{\text {ref }}+\delta T$ and $U=U_{\text {ref }}+\delta U$,

$$
\begin{aligned}
& m_{U_{\mathcal{R}}}^{2} \simeq(2 \pi)^{2}\left(U_{\mathcal{I}}+\frac{1}{\pi}\right)^{2} m_{3 / 2}^{2}+4 \pi\left(U_{\mathcal{I}}+\frac{1}{\pi}\right) m_{3 / 2}^{2}, \\
& m_{T_{\mathcal{R}}}^{2} \simeq 2\left(a T_{\mathcal{I}}\right)^{2} m_{3 / 2}^{2}
\end{aligned}
$$


for real part of moduli fields. By contrast, the mass matrices of imaginary parts are given in the basis $\left(U_{\mathcal{I}}, T_{\mathcal{I}}\right)$,

$$
\begin{aligned}
& m_{\mathcal{I}}^{2}=\left(\begin{array}{cc}
\frac{\partial_{U_{\mathcal{I}}} \partial_{U_{\mathcal{I}}} V}{2 K_{U \bar{U}}} & \frac{\partial_{U_{\mathcal{I}}} \partial_{T_{\mathcal{I}}} V}{2 \sqrt{K_{U \bar{U}}} \sqrt{K_{T \bar{T}}}} \\
\frac{\partial_{U_{\mathcal{I}} \partial_{T_{\mathcal{I}}} V}}{2 \sqrt{K_{U \bar{U}}} \sqrt{K_{T \bar{T}}}} & \frac{\partial_{T_{\mathcal{I}}} \partial_{T_{\mathcal{I}}} V}{2 K_{T \bar{T}}}
\end{array}\right) \\
& \simeq\left(\begin{array}{cc}
(2 \pi)^{2} U_{\mathcal{I}}^{2}\left(1+\frac{3}{2 K_{U_{\mathcal{I}} U_{\mathcal{I}}}} \frac{\partial_{U_{\mathcal{I}}} \partial_{U_{\mathcal{I}}} P}{P}\right) & \frac{3}{2 \sqrt{K_{U \bar{U}}} \sqrt{K_{T \bar{T}}}}\left[\frac{\partial_{T_{\mathcal{I}}} \partial_{U_{\mathcal{I}}} P}{P}+\frac{2}{3} K_{T_{\mathcal{I}}} \partial_{U_{\mathcal{I}}} \ln (P)\right] \\
\frac{3}{2 \sqrt{K_{U \bar{U}}} \sqrt{K_{T \bar{T}}}}\left[\frac{\partial_{T_{\mathcal{I}}} \partial_{U_{\mathcal{I}}} P}{P}+\frac{2}{3} K_{T_{\mathcal{I}}} \partial_{U_{\mathcal{I}}} \ln (P)\right] & 2\left(a T_{\mathcal{I}}\right)^{2}
\end{array}\right) m_{3 / 2}^{2},
\end{aligned}
$$

which shows that when the derivatives of $\mathcal{P}$ with respect to the moduli fields are of $\mathcal{O}(\mathcal{P})$, the mass squared of moduli fields can be positive in the limit of $a T_{\mathcal{I}} \gg 1$. According to it, $F$-terms of moduli fields are

$$
\begin{aligned}
\left\langle\frac{F^{T}}{T-\bar{T}}\right\rangle & \simeq \frac{1}{a T_{\mathcal{I}}} m_{3 / 2}\left[\left(\frac{5}{2}-i T_{\mathcal{I}} \partial_{T} \ln (\mathcal{P})\right)+\frac{24\left(\partial_{U} \ln \mathcal{P}\right)\left(\left(\partial_{T} \partial_{U} P\right) / P+i \frac{1}{T_{\mathcal{I}}} \partial_{U} \ln (\mathcal{P})\right)}{T_{\mathcal{I}}\left(K^{U \bar{U}}\left(K_{U_{\mathcal{I}} U_{\mathcal{I}}}\right)^{2}-16 K_{U U}\left(\partial_{U} \partial_{U} P\right) / P\right)}\right], \\
\left\langle\frac{F^{U}}{U-\bar{U}}\right\rangle & \simeq 12 \pi \frac{-i \partial_{U} \ln \mathcal{P}}{K^{U \bar{U}}\left(K_{U_{\mathcal{I}} U_{\mathcal{I}}}\right)^{2}-16 K_{U U}-24\left(\partial_{U} \partial_{U} P\right) / P} m_{3 / 2} .
\end{aligned}
$$

It turns out that the complex structure modulus has a sizable $F$-term, although it depends on the details of the uplifting sector.

Open Access. This article is distributed under the terms of the Creative Commons Attribution License (CC-BY 4.0), which permits any use, distribution and reproduction in any medium, provided the original author(s) and source are credited.

\section{References}

[1] K. Choi, A. Falkowski, H.P. Nilles, M. Olechowski and S. Pokorski, Stability of flux compactifications and the pattern of supersymmetry breaking, JHEP 11 (2004) 076 [hep-th/0411066] [INSPIRE].

[2] K. Choi, A. Falkowski, H.P. Nilles and M. Olechowski, Soft supersymmetry breaking in KKLT flux compactification, Nucl. Phys. B 718 (2005) 113 [hep-th/0503216] [INSPIRE].

[3] K. Choi, K.S. Jeong and K.-i. Okumura, Phenomenology of mixed modulus-anomaly mediation in fluxed string compactifications and brane models, JHEP 09 (2005) 039 [hep-ph/0504037] [INSPIRE].

[4] M. Endo, M. Yamaguchi and K. Yoshioka, A Bottom-up approach to moduli dynamics in heavy gravitino scenario: Superpotential, soft terms and sparticle mass spectrum, Phys. Rev. D 72 (2005) 015004 [hep-ph/0504036] [inSPIRE].

[5] V.S. Kaplunovsky and J. Louis, Model independent analysis of soft terms in effective supergravity and in string theory, Phys. Lett. B 306 (1993) 269 [hep-th/9303040] [INSPIRE].

[6] A. Brignole, L.E. Ibáñez and C. Muñoz, Towards a theory of soft terms for the supersymmetric Standard Model, Nucl. Phys. B 422 (1994) 125 [Erratum ibid. B 436 (1995) 747] [hep-ph/9308271] [INSPIRE]. 
[7] T. Kobayashi, D. Suematsu, K. Yamada and Y. Yamagishi, Nonuniversal soft scalar masses in superstring theories, Phys. Lett. B 348 (1995) 402 [hep-ph/9408322] [INSPIRE].

[8] L.E. Ibáñez, C. Muñoz and S. Rigolin, Aspect of type-I string phenomenology, Nucl. Phys. B 553 (1999) 43 [hep-ph/9812397] [INSPIRE].

[9] L. Randall and R. Sundrum, Out of this world supersymmetry breaking, Nucl. Phys. B 557 (1999) 79 [hep-th/9810155] [inSPIRE].

[10] G.F. Giudice, M.A. Luty, H. Murayama and R. Rattazzi, Gaugino mass without singlets, JHEP 12 (1998) 027 [hep-ph/9810442] [INSPIRE].

[11] S. Kachru, R. Kallosh, A.D. Linde and S.P. Trivedi, de Sitter vacua in string theory, Phys. Rev. D 68 (2003) 046005 [hep-th/0301240] [INSPIRE].

[12] K. Choi, K.S. Jeong, T. Kobayashi and K.-i. Okumura, Little SUSY hierarchy in mixed modulus-anomaly mediation, Phys. Lett. B 633 (2006) 355 [hep-ph/0508029] [INSPIRE].

[13] R. Kitano and Y. Nomura, A Solution to the supersymmetric fine-tuning problem within the MSSM, Phys. Lett. B 631 (2005) 58 [hep-ph/0509039] [INSPIRE].

[14] K. Choi, K.S. Jeong, T. Kobayashi and K.-i. Okumura, TeV Scale Mirage Mediation and Natural Little SUSY Hierarchy, Phys. Rev. D 75 (2007) 095012 [hep-ph/0612258] [InSPIRE].

[15] T. Kobayashi, H. Makino, K.-i. Okumura, T. Shimomura and T. Takahashi, TeV scale mirage mediation in NMSSM, JHEP 01 (2013) 081 [arXiv:1204.3561] [INSPIRE].

[16] K. Hagimoto, T. Kobayashi, H. Makino, K.-i. Okumura and T. Shimomura, Phenomenology of NMSSM in TeV scale mirage mediation, JHEP 02 (2016) 089 [arXiv:1509.05327] [INSPIRE].

[17] M. Asano and T. Higaki, Natural supersymmetric spectrum in mirage mediation, Phys. Rev. D 86 (2012) 035020 [arXiv: 1204.0508] [INSPIRE].

[18] M. Endo, K. Hamaguchi and F. Takahashi, Moduli-induced gravitino problem, Phys. Rev. Lett. 96 (2006) 211301 [hep-ph/0602061] [INSPIRE].

[19] S. Nakamura and M. Yamaguchi, Gravitino production from heavy moduli decay and cosmological moduli problem revived, Phys. Lett. B 638 (2006) 389 [hep-ph/0602081] [INSPIRE].

[20] M. Dine, R. Kitano, A. Morisse and Y. Shirman, Moduli decays and gravitinos, Phys. Rev. D 73 (2006) 123518 [hep-ph/0604140] [INSPIRE].

[21] D.H. Lyth and E.D. Stewart, Cosmology with a TeV mass GUT Higgs, Phys. Rev. Lett. 75 (1995) 201 [hep-ph/9502417] [INSPIRE].

[22] D.H. Lyth and E.D. Stewart, Thermal inflation and the moduli problem, Phys. Rev. D 53 (1996) 1784 [hep-ph/9510204] [INSPIRE].

[23] M. Fujii and K. Hamaguchi, Higgsino and wino dark matter from Q ball decay, Phys. Lett. B 525 (2002) 143 [hep-ph/0110072] [INSPIRE].

[24] H. Hattori, T. Kobayashi, N. Omoto and O. Seto, Entropy production by domain wall decay in the NMSSM, Phys. Rev. D 92 (2015) 103518 [arXiv:1510.03595] [INSPIRE].

[25] S. Nakamura, K.-i. Okumura and M. Yamaguchi, Axionic Mirage Mediation, Phys. Rev. D 77 (2008) 115027 [arXiv:0803.3725] [InSPIRE]. 
[26] A.D. Linde, Relaxing the cosmological moduli problem, Phys. Rev. D 53 (1996) 4129 [hep-th/9601083] [INSPIRE].

[27] K. Nakayama, F. Takahashi and T.T. Yanagida, On the Adiabatic Solution to the Polonyi/Moduli Problem, Phys. Rev. D 84 (2011) 123523 [arXiv:1109.2073] [InSPIRE].

[28] V. Balasubramanian, P. Berglund, J.P. Conlon and F. Quevedo, Systematics of moduli stabilisation in Calabi-Yau flux compactifications, JHEP 03 (2005) 007 [hep-th/0502058] [INSPIRE].

[29] J.P. Conlon, F. Quevedo and K. Suruliz, Large-volume flux compactifications: Moduli spectrum and D3/D7 soft supersymmetry breaking, JHEP 08 (2005) 007 [hep-th/0505076] [INSPIRE].

[30] S.B. Giddings, S. Kachru and J. Polchinski, Hierarchies from fluxes in string compactifications, Phys. Rev. D 66 (2002) 106006 [hep-th/0105097] [INSPIRE].

[31] S. Gukov, C. Vafa and E. Witten, CFT's from Calabi-Yau four folds, Nucl. Phys. B 584 (2000) 69 [Erratum ibid. B 608 (2001) 477] [hep-th/9906070] [INSPIRE].

[32] N.V. Krasnikov, On Supersymmetry Breaking in Superstring Theories, Phys. Lett. B 193 (1987) 37 [INSPIRE].

[33] L.J. Dixon, Supersymmetry Breaking In String Theory, SLAC-PUB-5229 (1990) [C90-01-03] [INSPIRE].

[34] O. Lebedev, H.P. Nilles and M. Ratz, de Sitter vacua from matter superpotentials, Phys. Lett. B 636 (2006) 126 [hep-th/0603047] [INSPIRE].

[35] E. Dudas, C. Papineau and S. Pokorski, Moduli stabilization and uplifting with dynamically generated F-terms, JHEP 02 (2007) 028 [hep-th/0610297] [INSPIRE].

[36] H. Abe, T. Higaki, T. Kobayashi and Y. Omura, Moduli stabilization, F-term uplifting and soft supersymmetry breaking terms, Phys. Rev. D 75 (2007) 025019 [hep-th/0611024] [INSPIRE].

[37] R. Kallosh and A.D. Linde, O'KKLT, JHEP 02 (2007) 002 [hep-th/0611183] [InSPIRE].

[38] H. Abe, T. Higaki and T. Kobayashi, More about F-term uplifting, Phys. Rev. D 76 (2007) 105003 [arXiv:0707.2671] [INSPIRE].

[39] T. Moroi and L. Randall, Wino cold dark matter from anomaly mediated SUSY breaking, Nucl. Phys. B 570 (2000) 455 [hep-ph/9906527] [INSPIRE].

[40] M. Kawasaki, K. Kohri and T. Moroi, Big-Bang nucleosynthesis and hadronic decay of long-lived massive particles, Phys. Rev. D 71 (2005) 083502 [astro-ph/0408426] [INSPIRE].

[41] M. Kawasaki, K. Kohri and T. Moroi, Hadronic decay of late-decaying particles and Big-Bang Nucleosynthesis, Phys. Lett. B 625 (2005) 7 [astro-ph/0402490] [InSPIRE].

[42] K. Kohri, T. Moroi and A. Yotsuyanagi, Big-bang nucleosynthesis with unstable gravitino and upper bound on the reheating temperature, Phys. Rev. D 73 (2006) 123511 [hep-ph/0507245] [INSPIRE].

[43] K.A. Olive and M. Srednicki, New Limits on Parameters of the Supersymmetric Standard Model from Cosmology, Phys. Lett. B 230 (1989) 78 [inSPIRE].

[44] Planck collaboration, P.A.R. Ade et al., Planck 2013 results. XVI. Cosmological parameters, Astron. Astrophys. 571 (2014) A16 [arXiv:1303.5076] [INSPIRE]. 
[45] Planck collaboration, P.A.R. Ade et al., Planck 2015 results. XX. Constraints on inflation, arXiv: 1502.02114 [INSPIRE].

[46] J.P. Conlon, The QCD axion and moduli stabilisation, JHEP 05 (2006) 078 [hep-th/0602233] [INSPIRE].

[47] M. Kawasaki, F. Takahashi and T.T. Yanagida, Gravitino overproduction in inflaton decay, Phys. Lett. B 638 (2006) 8 [hep-ph/0603265] [INSPIRE].

[48] K. Hori and C. Vafa, Mirror symmetry, hep-th/0002222 [INSPIRE].

[49] L.J. Dixon, V. Kaplunovsky and J. Louis, Moduli dependence of string loop corrections to gauge coupling constants, Nucl. Phys. B 355 (1991) 649 [INSPIRE].

[50] D. Lüst and S. Stieberger, Gauge threshold corrections in intersecting brane world models, Fortsch. Phys. 55 (2007) 427 [hep-th/0302221] [INSPIRE].

[51] S. Hosono, A. Klemm, S. Theisen and S.-T. Yau, Mirror symmetry, mirror map and applications to Calabi-Yau hypersurfaces, Commun. Math. Phys. 167 (1995) 301 [hep-th/9308122] [INSPIRE].

[52] S. Hosono, A. Klemm, S. Theisen and S.-T. Yau, Mirror symmetry, mirror map and applications to complete intersection Calabi-Yau spaces, Nucl. Phys. B 433 (1995) 501 [hep-th/9406055] [INSPIRE].

[53] K.A. Intriligator, N. Seiberg and D. Shih, Dynamical SUSY breaking in meta-stable vacua, JHEP 04 (2006) 021 [hep-th/0602239] [INSPIRE].

[54] T. Kobayashi, A. Oikawa and H. Otsuka, New potentials for string axion inflation, Phys. Rev. D 93 (2016) 083508 [arXiv: 1510.08768] [InSPIRE].

[55] M. Kawasaki, T. Moroi and T. Yanagida, Can decaying particles raise the upper bound on the Peccei-Quinn scale?, Phys. Lett. B 383 (1996) 313 [hep-ph/9510461] [INSPIRE].

[56] I. Affleck and M. Dine, A New Mechanism for Baryogenesis, Nucl. Phys. B 249 (1985) 361 [INSPIRE].

[57] M. Dine, L. Randall and S.D. Thomas, Baryogenesis from flat directions of the supersymmetric standard model, Nucl. Phys. B 458 (1996) 291 [hep-ph/9507453] [INSPIRE]. 